\title{
IMPLICACIÓN DEL ALUMNADO EN LA VALORACIÓN DE SU SATISFACCIÓN CON LAS WEBS DIDÁCTICAS
}

\section{IMPLICATION OF THE STUDENT BODY IN THE APPRAISAL OF HIS SATISFACTION WITH THE USE OF DIDACTIC WEBSITES}

\author{
Ana Belén Mirete Ruiz; anabelen.mirete@um.es \\ Francisco Alberto García-Sánchez; fags@um.es

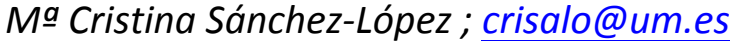 \\ Facultad de Educación. \\ Universidad de Murcia
}

\section{RESUMEN}

Presentamos el proceso y resultado del esfuerzo por implicar al alumnado universitario en la valoración de Web Didácticas. Hemos trabajado cooperativamente con alumnos y profesores, con el objetivo de conseguir un instrumento de evaluación ajustado a la percepción que ambos colectivos tienen del recurso. Nuestros resultados aportan un conjunto de ítems para valorar la satisfacción del alumnado con las webs didácticas y la comprobación de que ese recurso, desde una perspectiva didáctica, resulta muy bien valorado por el alumnado.

PALABRAS CLAVE: Web Didáctica; universidad; satisfacción; alumnado; investigación cooperativa.

\section{ABSTRACT}

We introduce the process and the results of an effort in order to implicate the universitary student body in the appraisal of their satisfaction about the use of the didactic webs. We have worked hand to hand with students and professors, with the aim to obtain an instrument of evaluation adjusted to the perception that both groups have of the resource. Our results provide a set of items to evaluate the satisfaction of the learners with the use of didactic websites and the test that this technological resource, oriented from a didactical view, is well valued by the student body.

KEY WORDS: Didactic Websites; university; satisfaction; student body; cooperative investigation. 


\section{INTRODUCCIÓN}

Los roles que se han venido desempeñando tradicionalmente por docentes y discentes están cambiando debido a las nuevas exigencias planteadas por el Espacio Europeo de Educación Superior (EEES). Entre ellas está la de potenciar la figura del profesor como facilitador del aprendizaje de sus alumnos. Una de sus tareas principales es potenciar en los estudiantes su papel de agentes activos, responsables de gestionar su ambiente de aprendizaje y conseguir que exista un trabajo constante y fluido (Benito \& Ovelar, 2005; Monedero, 2007; Salinas, 1997).

La combinación EEES y TIC nos ofrece la posibilidad de replantear y apoyar tanto la docencia teórica como las prácticas docentes. Contamos con nuevas herramientas con las que desarrollar metodologías más comprometidas con el modelo educativo a desarrollar. Ahora bien, la mera introducción de las TICS en el aula, o la simple generación de recursos que las empleen como base, no es suficiente. La prioridad debería ser crear materiales pedagógicamente adecuados y no centrarse, exclusivamente, en que su diseño sea técnicamente perfecto, ya que su valor radica en que estos resulten significativos y útiles para los alumnos que las van a emplear.

Para lograr ese alumno más autónomo, gestor de su propio proceso de enseñanzaaprendizaje, el docente necesita un soporte tecnológico adecuado en el que ofrecer al estudiante aquello que considera necesario para que construya su conocimiento y supere la asignatura (desde documentos científicos y técnicos, en diferentes posibles formatos y enlaces de interés, hasta orientaciones tutoriales sobre "para qué» y "cómo» utilizar ese material). Además, se trata también de introducir nuevas modalidades de aprender, crear oportunidades novedosas de acceder al conocimiento y abrir una puerta a la realidad tecnológica (Chiecher, Donolo \& Rinaudo, 2005).

Una herramienta que da respuesta a varias de las necesidades surgidas en estos momentos de cambio son las Webs Didácticas de asignatura. Las definimos, desde nuestra perspectiva docente universitaria, como páginas web pensadas, diseñadas y destinadas a facilitar los procesos de enseñanza y aprendizaje, a la vez que sirve de guía al alumno (Area, 2003; Bueno \& Gil, 2007; García-Sánchez \& Martínez-Segura, 2009; Marqués, 2005; Mur \& Serrano, 2002). La Web Didáctica es ese espacio común, donde el docente encuentra un lugar para compartir con el alumno todo aquello que puede repercutir en el proceso de gestión de su aprendizaje. Allí podemos encontrar la descripción de las competencias a desarrollar, los contenidos y las actividades con las que se trabajarán dichas competencias, posibles itinerarios de aprendizaje, recursos disponibles desde la misma Web o en otras fuentes, orientaciones tutoriales para el estudio y el aprendizaje, etc.

Las decisiones que se adopten en la elaboración y estructura de la página Web, tanto técnicas como educativas, van ha incidir directamente en el interés y la motivación que los estudiantes van a desarrollar por esta herramienta. Los recursos educativos sólo adquieren verdadero sentido cuando están plenamente integrados en el sistema de enseñanza (Monedero, 2007), y para ello es preciso que el estudiante los acepte dentro de su proceso de aprendizaje, haciendo de ellos herramientas básicas de su trabajo formativo.

Son varios los estudios realizados sobre enseñanza virtual que buscan comprender la percepción del alumnado con este tipo de metodología (Chandra \& Fisher, 2009; Cabero, Llorente \& Puentes, 2010; Chiecher, Donolo \& Rinaudo, 2005; DeBourgh, 1999; Hinojo, Aznar \& Cáceres, 2009; Lim, Morris \& Kupritz, 2007; Recio \& Cabero, 2005; Shahzad \& Khan, 
2010; Tesone \& Ricci, 2008; Villa, 2005). Pero la satisfacción del estudiante es un concepto complejo de delimitar, lo que nos ha llevado a considerar que la participación de ese alumno en la elaboración de un instrumento de medida, puede ayudarnos a conocer en qué radica su verdadera aceptación de estos recursos Web. El propio alumno nos puede facilitar información sobre qué aspectos, elementos, contenidos, presentaciones, etc., contribuyen a elevar ese nivel de satisfacción, tan difícil de valorar.

El objetivo que nos propusimos al iniciar este trabajo, por tanto, fue doble. Primeramente buscábamos elaborar un instrumento que nos facilitara recoger información sobre la percepción del estudiante y su nivel de satisfacción con el uso de webs didácticas en el aula. Pero cualquier innovación docente, va a estar diseñada pensando en el estudiante, en sus características, limitaciones, potencialidades y necesidades, por lo que su opinión sobre estas innovaciones va a ser fundamental (Chandra \& Fisher, 2009). Por este motivo, nos planteamos como segundo objetivo, no menos importante que el primero, desarrollar una dinámica de trabajo cooperativo teniendo al alumno como referente y protagonista principal. De esta manera, inspirándonos en los postulados básicos de la investigación cooperativa o colaborativa (Bartolomé \& Anguera, 1990, Latorre, Del Rincón \& Arnal, 2003) cuya pretensión última es la toma de decisiones en busca de mejorar los recursos empleados en el aula $y$, en consecuencia, la práctica educativa, contamos con la participación del alumno que conoce las Webs Didácticas y las está empleando en su proceso de aprendizaje, y por tanto puede contribuir a la mejora del recurso aportando su perspectiva discente.

\section{MATERIAL Y MÉTODO}

\subsection{Participantes:}

Para la realización de este trabajo se ha utilizado una muestra total de 146 alumnos y 13 profesores, los cuales se han visto implicados en alguna de las distintas fases del procedimiento utilizado. En concreto, han participado en el estudio:

- 19 alumnas de cuarto curso de la Licenciatura de Pedagogía, las cuales empleaban Web Didáctica para el desarrollo de al menos una asignatura.

- 5 alumnas de la Diplomatura de Logopedia (2 de segundo curso y 3 de tercer curso), constituyeron el Grupo Focal de Discusión que delimitó la composición y estructura de la primera versión del cuestionario diseñado.

- 13 Profesores de la Universidad de Murcia (2 Catedráticos, 4 Titulares, 4 Ayudantes y 3 Asociados), constituyeron el grupo de expertos para enjuiciar el contenido del instrumento diseñado. Todos estos profesores han participado en distintos Proyectos de Innovación Educativa en los últimos años, relacionados con experiencias vinculadas al diseño e implementación de Webs Didácticas. En el momento de valorar el cuestionario diseñado, todos ellos participaban en un proyecto de implementación de webs didácticas para apoyo de la docencia presencial financiado por el Vicerrectorado de Innovación y Convergencia Europea de la Universidad de Murcia.

- Un total de 122 alumnos de primer curso del Grado de Logopedia (91 alumnos), de tercer curso de la Diplomatura de Magisterio especialidad de Educación Especial (19 alumnos) y de segundo curso de la Diplomatura de Educación Social 
(12 alumnos), a los cuales se aplicó el instrumento para el análisis de su satisfacción con la primera experiencia de uso de una Web didáctica de asignatura.

\subsection{Procedimiento:}

El procedimiento seguido constituye una adaptación de las fases que proponen Cohen \& Manion (1990), buscando conseguir los criterios de calidad que se marcan para la investigación cualitativa (credibilidad, confirmabilidad y transferibilidad o aplicabilidad). De acuerdo con ello, nuestro trabajo se ha desarrollado en seis fases:

1a Fase. Planteamiento del problema. Revisión bibliográfica de estudios centrados en la motivación, percepción e interés que suscitan, en el estudiante universitario, el uso de recursos Web y metodologías basadas en blended learnig y metodologías similares. La revisión de estos trabajos nos facilitó y guió para el diseño de una plantilla de temas a tratar con la que iniciar la primera recogida de información con el alumnado. Presentar la plantilla tenía como propósito servir de herramienta para centrar al estudiante en el objetivo del estudio.

2a Fase. Aplicación de la técnica grupal «Bola de Nieve», una técnica de muestreo que aplicada a la recogida de información, ofrece la ventaja de acumular información valiosa para la detección de problemas y/o necesidades de grupos con intereses comunes. Se aplicó a un grupo compuesto por 19 alumnas de cuarto curso de la titulación de Pedagogía, con experiencias repetidas en uso de webs didácticas, la cual variaba de una alumna a otra, oscilando entre 1 y 3 asignaturas cursadas con este recurso, con una media de 2.2 asignaturas para todo el grupo de alumnas. Se partió de la plantilla de temas a tratar elaborada en la fase anterior, y para su cumplimentación se realizó una pequeña introducción de la actividad a realizar y el motivo que nos llevaba a aplicarla.

3a Fase. Grupo focal de discusión con alumnos. A partir de los resultados obtenidos en la aplicación de la técnica grupal, se llevó a cabo un análisis de la información en un Grupo Focal de discusión constituido por 5 alumnas. Su experiencia acumulada en el uso de webs didácticas, junto con la vivida en el momento de desarrollo de los grupos de discusión, las capacitaban para el análisis crítico y reflexión hacia los motivos que podían generar su propio nivel de satisfacción/insatisfacción con las herramientas Web. La característica principal de los grupos focales es que se trata de un grupo de trabajo que tiene una tarea concreta y unos objetivos que lograr en torno a un tema específico que admite diferentes perspectivas o puntos de vista, y por ello requieren de distintos enfoques que puedan ofrecerse desde experiencias e intereses, igualmente distintos. A través del diálogo, la interacción y el intercambio de opiniones, logran enriquecer y complementar la percepción de la realidad, con el objetivo de alcanzar una visión compartida y bien fundamentada por las aportaciones de todos los miembros del grupo (Martínez-Miguélez, 2004), motivo por el que se seleccionó este procedimiento para nuestro estudio.

4a Fase. Dado que el propósito final era el diseño y construcción del instrumento de evaluación de la satisfacción del alumnado, se concertaron varias reuniones con los objetivos de: 1) diseñar el formato del cuestionario; 2) identificar y analizar los bloques de ítems y la relevancia de los mismos en la medición del índice de satisfacción; y 3) redactar los ítems que compondrían la primera versión del cuestionario. 
5a Fase. Revisión, discusión y propuestas de mejora a través de un procedimiento de «juicio de expertos». Elaborada la versión inicial del cuestionario, en esta fase se sometió a revisión por un grupo de 13 profesores de universidad implicados en proyectos de Innovación Educativa $y$ en el diseño e implementación de webs didácticas para asignaturas universitarias. Con tal fin se les facilitó una copia del instrumento de valoración a cada uno de los miembros del grupo para su estudio. Posteriormente, se dio la oportunidad de discutir su contenido y los aspectos formales, tanto a través de una reunión grupal, como a través de la presentación de apreciaciones a nivel particular. Las apreciaciones que pudieron ser consensuadas o se consideraron pertinentes fueron incorporadas como mejoras en la segunda versión del cuestionario realizado.

6a Fase. Aplicación del cuestionario. Esta aplicación se llevó a cabo al finalizar el curso 2009/10, a un total de 122 alumnos, de tres titulaciones, que cursaban por primera vez una asignatura universitaria con Web didáctica.

\section{RESULTADOS}

El resultado de la primera fase de nuestro procedimiento nos llevó a delimitar los núcleos de interés encontrados en la bibliografía consultada sobre satisfacción del alumno y que, aplicados a nuestro objeto de estudio, se concretaron en delimitar aspectos positivos, negativos y/o mejorables de la Web didáctica en torno a cuatro dimensiones: el desarrollo de la autonomía en el alumno, la calidad de la enseñanza, las características de las clases y el diseño de la asignatura.

La aplicación de la técnica grupal ofreció los primeros datos que sirvieron para el trabajo con el Grupo Focal de alumnos en la Fase III. La información que se obtuvo sobre los cuatro núcleos de interés trabajados queda resumida en la Tabla 1.

\begin{tabular}{|c|c|c|}
\hline Aspectos Positivos & Aspectos Negativos & Aspectos Mejorables \\
\hline $\begin{array}{l}\text { Acceso a los contenidos } \\
\text { de la asignatura } \\
\text { Organización y } \\
\text { Estructuración } \\
\text { Fomento de la } \\
\text { autonomía } \\
\text { Facilitador de la } \\
\text { comunicación } \\
\text { Disponibilidad de mayor } \\
\text { volumen de } \\
\text { información } \\
\text { Otros (Motivación, } \\
\text { aprendizaje del recurso, } \\
\text { clases dinámicas) }\end{array}$ & $\begin{array}{l}\text { Necesidad de formación } \\
\text { para el uso del recurso } \\
\text { Dificultad por acceso a } \\
\text { través de Internet } \\
\text { Falta de control } \\
\text { docente sobre el } \\
\text { proceso de aprendizaje } \\
\text { Necesidad de } \\
\text { explicación teórica de } \\
\text { los contenidos } \\
\text { Requiere más tiempo } \\
\text { de dedicación y } \\
\text { atención } \\
\text { No se emplea en el aula }\end{array}$ & $\begin{array}{l}\text { Formación en el manejo } \\
\text { del recurso } \\
\text { Diseño, organización y } \\
\text { planificación de la Web } \\
\text { Integración del recurso } \\
\text { en el aula } \\
\text { Inclusión de materiales } \\
\text { y actividades } \\
\text { complementarias }\end{array}$ \\
\hline
\end{tabular}


De acuerdo a estos resultados, lo que más valoran los alumnos en la Web Didáctica es la posibilidad de acceso a los contenidos de la asignatura y que su organización y estructura se corresponda a la de la asignatura. Por el contrario, lo que peor valoran es que haya necesidad de una formación específica para usar el recurso y las dificultades encontradas en el acceso a Internet. Por último, los alumnos entienden como especialmente importante, en la práctica que han tenido con Webs Didácticas, recibir una formación en el manejo del recurso y que el diseño, organización y planificación de la Web estén cuidados.

La información obtenida constituyó la base de trabajo del Grupo Focal de Discusión de alumnos. Los datos se estructuraron en cinco bloques: Utilidad; Acceso y navegabilidad; Formación para su uso; Contenidos y acceso a la asignatura; y Recursos complementarios. Estos bloques, a su vez, ayudaron a la posterior redacción de los ítems. El grupo de trabajo tomó decisiones sobre los contenidos de los ítems a incluir, el diseño del cuestionario, el modo de presentación y redacción de los ítems y sus opciones de respuesta. Entre otras cuestiones, se determinó la estructura de ítems con tres propuestas de respuesta cerrada en torno a diferentes grados de satisfacción. Esta decisión se adoptó por plantearse como uno de los mayores hándicaps, a la hora de proponer un cuestionario a los estudiantes, la falta de implicación de éstos en la respuesta ofrecida. Según los propios alumnos, este hecho se favorece al proponer una relación de ítems con valoración numérica, ante la cual el alumnado tiende a automatizar un patrón de respuesta, reforzado por su mayor o menor simpatía con el profesorado y con poca reflexión sobre la cuestión planteada. La misma razón guió también a la presentación de las respuesta de forma aleatoria, evitando así la posible instauración de un patrón de respuesta fijo. De esta forma se pretendía obligar a la lectura detenida de los tres enunciados propuestos y a la selección de aquel que se ajuste a su verdadero sentimiento de satisfacción.

El primer borrador del instrumento de valoración de satisfacción es el resultado del proceso seguido durante cuatro sesiones de trabajo, donde las estudiantes participantes fueron las encargadas de aportar nuevos puntos de vista, tomar decisiones y diseñar el cuestionario. Como continuación de ese trabajo, se delimitó un tiempo para la revisión individual del cuestionario; tras el cual se llevó a cabo una última puesta en común y se introdujeron algunas mejoras en los ítems que podían llevar a confusión al alumno.

Con la nueva versión del instrumento que surgió del trabajo del Grupo Focal, se inició el proceso de someterlo a evaluación por parte del grupo de profesores. Esta fase finalizó con la inclusión de las mejoras y propuestas realizadas por este nuevo grupo de trabajo, tanto de manera individual como en la reunión final mantenida a tal efecto. Todo ello dio lugar a la versión final del cuestionario de valoración.

Finalmente, el instrumento diseñado para valorar la satisfacción del estudiante universitario con el uso de webs didácticas quedó configurado por un total de 14 ítems. Diez de estos ítems tienen tres opciones cerradas de respuesta, de las cuales se pedía al alumno sólo eligiese una. Estas opciones de respuesta fueron presentadas en orden aleatorio, pero intentaban graduar la respuesta entre menos y más satisfecho, con un enunciado intermedio. A estos ítems se suma uno más, con solo dos opciones de respuesta. Los otros tres ítems del cuestionario pedían al alumno una valoración, en una escala de 0 a 3 puntos, el grado de satisfacción general con la organización y disponibilidad del contenido en la Web didáctica y con el propio uso dado a la Web didáctica.

La versión final del instrumento se aplicó a una muestra de 122 alumnos que utilizaban por primera vez una Web didáctica de asignatura en sus estudios universitarios. Todas las 
asignaturas implicadas eran impartidas por profesores diferentes y contaban con webs didácticas que, aunque distintas en diseño y estructura, pretendían objetivos similares.

Los datos obtenidos con la aplicación del cuestionario nos muestran cómo el estudiante expresa un alto nivel de satisfacción general (Tabla 2). Su satisfacción es alta con las posibilidades que ofrece la Web didáctica en relación a la organización de los contenidos y disponibilidad de los mismos, puntuando con una media de 2,4 y 2,54 puntos sobre 3 en los ítems correspondientes. Así mismo, cuando se pregunta sobre el nivel general de satisfacción con el uso de la Web didáctica, los resultados alcanzan una puntuación media de 2,53 sobre 3 . Ello nos indica un grado de satisfacción elevado ante el recurso y con la posibilidad de contar con un lugar desde el cual poder disponer de los contenidos de la asignatura organizados por el profesor.

\begin{tabular}{l|r|r|r|r|}
\hline Ítem & N. Válido & Media & S.D. \\
\hline Mi grado de satisfacción con la organización de contenidos & 109 & 2,40 & 0,54 \\
\hline $\begin{array}{l}\text { Mi grado de satisfacción con la disponibilidad de contenidos } \\
\text { Y en general, mi grado de satisfacción el uso de Web }\end{array}$ & 106 & 2,54 & 0,55 \\
\hline $\begin{array}{l}\text { Didácticas } \\
\text { Tabla 2. Media y desviación típica de los tres ítems del cuestionario que preguntaban sobre nivel de satisfacción } \\
\text { general (con posibilidad de valoración entre O y 3 puntos). }\end{array}$
\end{tabular}

Los datos que presentamos a continuación (Tabla 3) recogen los resultados de las distintas opciones de los ítems del cuestionario y ofrecen información relevante sobre ese nivel de satisfacción que buscamos medir.

\begin{tabular}{|c|c|c|}
\hline $\begin{array}{l}\text { 1. La Web-Didáctica me resulta útil para mi aprendizaje en las asignaturas } \\
\text { que la emplean. }\end{array}$ & Frec. & $\%$ \\
\hline Siempre & 88 & 72.7 \\
\hline No le encuentro utilidad alguna & 1 & .8 \\
\hline A Veces & 32 & 26.4 \\
\hline 2. La Web Didáctica me ha sido útil en el aprendizaje para: & Frec. & $\%$ \\
\hline Únicamente la preparación del examen & 8 & 6.6 \\
\hline $\begin{array}{l}\text { Tanto para la superación de la asignatura como para mi desarrollo personal y } \\
\text { profesional }\end{array}$ & 52 & 43.0 \\
\hline Para ampliar los contenidos de la asignatura & 61 & 50.4 \\
\hline 3. El tiempo que requiere el uso de este tipo de recursos me parece: & Frec. & $\%$ \\
\hline Excesivo & 27 & 22.7 \\
\hline Adecuado & 91 & 77.3 \\
\hline 4. Navegar por la página me ha resultado: & Frec. & $\%$ \\
\hline Muy complicado y me ha dificultado mucho la tarea & 7 & 5.9 \\
\hline Difícil, y en ocasiones no he encontrado lo que busco & 32 & 26.9 \\
\hline Fácil y siempre he conseguido mi objetivo & 80 & 67.2 \\
\hline 5. El acceso a través de la red hace que: & Frec. & $\%$ \\
\hline Me parezca bien, aunque encuentro algunos obstáculos pa & 62 & 51.7 \\
\hline
\end{tabular}


Esté muy descontento por las dificultades que plantea la disponibilidad de Internet

Esté muy satisfecho porque puedo acceder desde cualquier lugar

6. La formación básica que tenemos en el uso de recursos Web hace que el uso de la Web-Didáctica

No sea un problema porque todos sabemos manejar NNTT

Sea un obstáculo grande, que hace que la Web pierda utilidad

Sea un obstáculo, pero superable con la práctica

7. La formación inicial que ha ofrecido el profesor sobre el manejo del recurso Web empleado:

Me resultó aburrida porque pienso que no era necesaria

Hizo que encontrase algunas cosas útiles, pero ya tenía los conocimientos básicos

Me gustó mucho porque pienso que es imprescindible

$58 \quad 49.6$

8. Prefiero que las webs didácticas:

Se sigan usando en aquellas asignaturas que ya se emplean, pero que no se generalice a todas

No se usen y dejen de emplearse en aquellas asignaturas en las que ya se hace

Se empleen en todas las asignaturas

9. El poder trabajar un mismo contenido con diferentes materiales (textos, power point, vídeos, etc.) desde la Web Didáctica, hace que:

Me pierda entre los materiales sin llegar a sacar la esencia del contenido

Los vea como recursos complementarios que a veces me pueden servir

10. El grado de autonomía en el control del aprendizaje que me permite el uso de la Web didáctica:

No me parece bien porque puede haber dejadez por parte del alumno

No me afecta porque sigo el ritmo de las clases teóricas

Me parece bien porque permite avanzar a cada alumno según su ritmo individual

\section{Tener la asignatura estructurada cronológicamente me resulta:}

Frec. \%

Estresante porque me obliga a organizarme en función a la asignatura

Muy interesante porque me ayuda a organizar la asignatura

Indiferente porque voy al día y sigo el ritmo de las clases teóricas

\section{Tabla 3. Frecuencias y porcentajes alcanzados por las distintas opciones de los ítems del cuestionario aplicado.}

Como podemos apreciar, un $72.7 \%$ del alumnado encuestado señala que la Web didáctica siempre es útil para el aprendizaje (ítem 1), bien sea (como se observa en el ítem 2) para ampliar los contenidos (opción elegida por el 50,4\% de los encuestados) o para superar la asignatura y conseguir un desarrollo personal y profesional (43,0\% de los encuestados). Siendo consecuentes con estos datos, vemos en el ítem 8 que el alumnado expresa su deseo de que siga utilizándose el recurso en la asignatura en la que lo han empleado $(43,7 \%$ de los alumnos) e incluso que se generalice su uso $(50,4 \%)$. 
En los ítems 9, 10 y 11 se valora la utilidad de las webs didácticas en diversos aspectos. Para trabajar los contenidos, se ve útil fundamentalmente ya que es una herramienta que ayuda a asimilar de forma integral los contenidos (55,8\%); ya que permite avanzar a cada alumno según su ritmo individual ( $72,6 \%$ de los alumnos); y su estructuración cronológica resulta muy interesante para ayudar a organizar la asignatura $(74,8 \%)$.

Uno de los aspectos que se revelaron de gran importancia, tanto en el grupo inicial como en el grupo focal de alumnos con los que se trabajó el instrumento de recogida de información, eran los problemas de tiempo, la navegabilidad y el acceso a la Web a través de Internet. Sin embargo, un 77,3\% del alumnado encuestado considera que el tiempo requerido por el uso del recurso es adecuado (ítem 3 ). Por otro lado, los datos nos muestran cómo ese acceso a la Web didáctica a través de la red no representa un problema para el $41,7 \%$ de los alumnos, que indican estar muy satisfechos ya que pueden acceder a la información desde cualquier lugar; mientras que otro $51,7 \%$ de los alumnos indican que les parece bien el acceso a la información a través de la red, aunque hayan encontrado algunos problemas (ítem 5). En lo referente a la navegación por la página, un $67,2 \%$ de los alumnos lo han encontrado fácil y siempre han conseguido su objetivo y sólo un $5,9 \%$ de estos alumnos consideran que ha sido muy complicado (ítem 4).

Los ítems 6 y 7 hacen alusión a la "formación en el uso de tecnologías» y cómo ha podido influir en la satisfacción del estudiante hacia la Web didáctica empleada. A pesar de haberse mostrado como una necesidad y demanda básica en la técnica grupal inicialmente empleada, los datos obtenidos en el ítem número 6 nos señalan que el 57,5\% del alumnado encuestado considera que tiene una formación adecuada en el manejo de nuevas tecnologías que le permite usar la Web didáctica sin problemas. De hecho, ningún alumno ve la posible falta de formación básica en TIC como un obstáculo insalvable. Además, se valora como satisfactoria o muy satisfactoria la formación inicial ofrecida por el profesorado (ítem 7).

\section{DISCUSIÓN}

Uno de los propósitos fundamentales de este trabajo ha sido desarrollar una herramienta que permita acercarnos a conocer el grado de satisfacción de los estudiantes ante el uso de las páginas web que se están empleando para el seguimiento de algunas asignaturas.

En los últimos años son muchos los recursos didácticos distribuidos en red que están surgiendo gracias a las posibilidades que brindan las TIC a la docencia universitaria. Para su valoración y estudio encontramos disponibles instrumentos de evaluación de materiales multimedia, recursos educativos virtuales, o de experiencias concretas desarrolladas con apoyo de las tecnologías, ya sean de e-learning o blended learning. Ahora bien, aún carecemos de herramientas de evaluación específicas sobre páginas web didácticas que nos aporten información válida con la que ir mejorando estos recursos, más aún si hablamos de la satisfacción que genera en el estudiante.

Tras repetidas experiencias de diseño e implementación de Webs Didácticas, hemos podido comprobar como la aceptación de las mismas por parte del alumnado difiere en función del grupo y la asignatura en la que se inserta. Por ello consideramos fundamental contar con un instrumento que aportase información, ya no de las cualidades técnicas de la página web, ni tampoco de la calidad de los recursos incluidos, sino de qué elementos son aquellos mejor 
valorados por el alumno, así como la manera en la que prefiere que le sean presentados o las posibilidades que les ofrecen, pudiendo identificar, de esta manera, los procesos que están dando mejores resultados educativos.

Por otra parte, uno de los logros más destacables de este trabajo ha sido la colaboración entre estudiantes e investigadores en la búsqueda de un objetivo común: diseñar y elaborar un instrumento que nos facilite información sobre ese nivel de satisfacción, tan difícil de cuantificar. De esta estrecha colaboración resulta el cuestionario que presentamos, el cual cubre altamente nuestras expectativas, ya que ha sido elaborado contando con la perspectiva del estudiante.

Tras la aplicación del cuestionario resultado de este trabajo, podemos afirmar que la valoración de las webs didácticas por parte del alumnado resulta muy satisfactoria, opinión que coincide con los datos presentados por Cabero, Llorente y Puentes (2010) en la alta valoración que realizan los estudiantes del entorno virtual empleado en un proceso de aprendizaje mezclado. Nuestro grupo de investigación ya había obtenido resultados similares en estudios previos realizados (García-Sánchez \& Martínez-Segura, 2009, GarcíaSánchez \& cols., 2010), pero en esta ocasión se han valorado aquellos aspectos que el propio alumnado ha considerado especialmente pertinentes e interesantes. Precisamente la importancia que se le ha otorgado en este estudio al punto de vista de los alumnos, para el propio desarrollo de la investigación, ha sido fundamental desde los primeros pasos. Han estado presentes durante el establecimiento de las ideas básicas, han sido especialmente relevantes en el diseño del instrumento elaborado para medir ese grado de satisfacción («su» grado de satisfacción), y han sido los que han generado los datos que finalmente analizamos.

Contrariamente a lo que muchas veces el profesorado piensa o alega, el alumnado no ve especiales problemas en el uso de las TICs, en el acceso a contenidos a través de Internet, o en la falta de preparación para utilizar este tipo de recursos. Obviamente, desarrollados en una cultura donde los medios audiovisuales y el ordenador están altamente integrados, entienden que tienen capacidad para aprovechar estos recursos y superar las dificultades iniciales que puedan encontrar. Faltaría saber si el profesorado opina igual antes esos mismos recursos.

En cuanto a las características de las webs didácticas que más se valoran, el alumnado destaca el hecho de que la herramienta le permita libertad para avanzar a su ritmo individual, la ayuda que significa para la organización de la asignatura al presentar una estructura cronológica y que permita una visión integral del contenido. Muy pocos alumnos ven en ella un recurso solo para preparar el examen, sino más bien para aprender, y este es precisamente uno de los objetivos marcados al entrar en el EEES. También son muy pocos los alumnos que piensan que una herramienta así puede contribuir a que se pierdan entre materiales sin llegar a sacar la esencia del contenido o que pueda favorecer la dejadez por parte del alumno.

Todos estos resultados nos impulsan a seguir defendiendo la Web didáctica de asignatura, estructurada con unos objetivos didácticos y desde la experiencia del profesor, como una herramienta especialmente útil para alcanzar las metas que nos plantea el EEES.

En este estudio se ha pretendido que en todo momento su desarrollo tuviera como referente al alumnado, sus impresiones, necesidades, problemas, etc. para poder avanzar y profundizar en el conocimiento de los problemas inmediatos y prácticos que afectan a su rol 
discente. Pero también hemos podido contar con la participación y colaboración de profesores que están desarrollando e implementado este tipo de recursos. La experiencia en el diseño, elaboración e implementación de webs didácticas, el conocimiento del funcionamiento del recurso en el aula y las impresiones causadas en los estudiantes como en el profesorado, resultan datos indispensables para acercarnos de una forma holística a un mejor conocimiento de estas herramientas, sus posibilidades y sus utilidades de cara a su integración en la docencia universitaria.

\section{REFERENCIAS}

AREA MOREIRA, M. (2003). Guía Didáctica. Internet en la docencia universitaria. Webs Docentes $y$ Aulas Virtuales. Disponible On-Line en http://cedus.cl/files/guiadidacticawebs.pdf [Consultado en Diciembre de 2010].

BARTOLOMÉ, M. \& ANGUERA, M.T. (1990). La investigación cooperativa: vía para la innovación en la Universidad. Barcelona: PPU.

BENITO, M. \& OVELAR, R. (2005). Impacto de las TIC y del proceso de convergencia al EEES en el profesorado universitario. Observatorio de e-learning. Universidad del País Vasco. Disponible On-Line

en: http://pulsar.ehu.es/pulsar/documentacion/informes_pulsar/Informe_PULSAR_Diciembre.p df. [Consultado en Diciembre de 2009].

BUENO, C. \& GIL, J.G. (2007). Web docente: estructura y procedimientos básicos de gestión eficaz. Revista Interuniversitaria de Formación del Profesorado, 21 (1), 37-50.

CABERO, J., LLORENTE, C. \& PUENTES, A. (2010). La satisfacción de los estudiantes en red en la formación semipresencial. Comunicar, 35, XVIII. 149-157.

CHANDRA, V. \& FISHER, D.L. (2009). Students' perceptions of a blended web-based learning environment. Learning Environ Res, 12, 31-44.

CHIECHER, A., DONOLO, D. \& RINAUDO, M.C. (2005). Percepciones del aprendizaje en contextos presenciales y virtuales. La perspectiva de los alumnos universitarios. RED. Revista de Educación a Distancia, 13, (V). Disponible On-Line en: http://www.um.es./ead/red/13/chiecher.pdf. [Consultado en Enero de 2010].

COHEN, M. \& MANION, L. (1990). Métodos de investigación educativa. Madrid: La Muralla.

DEBOURG, G.A. (1999). Tecnhology is the tool, teaching its the task: Student satisfaction in distance learning. Site 99: Society for Information Technology and Teacher Education International Conference. San Antonio, TX, Del 28 de febrero al 4 de marzo.

GARCÍA-SÁNCHEZ, F.A. \& MARTÍNEZ-SEGURA, M.J. (2009). WEB-docente y aprendizaje: una experiencia en el contexto de la convergencia al EEES. En R. Roig Vila (Coord.). Investigar desde un contexto educativo innovador (pp. 201-217). Alcoy: Marfil.

GARCÍA-SÁNCHEZ, F.A., MARTÍNEZ-SEGURA, M.J., MIRETE, A. \& MARTÍNEZ-JUÁREZ, M. (2010). Diseño de Webs-Home para asignaturas del Departamento de Métodos de Investigación y Diagnóstico en Educación. En Arnaiz, P., Hernández Abenza, L. y García Sanz, 
M.P. (Eds.). Experiencias de Innovación en la Universidad de Murcia durante 2009 (pp.115141). Murcia: Editum.

HINOJO, F.J., AZNAR, I. \& CÁRCELES, M.P. (2009). Percepciones del alumnado sobre el blended learning en la universidad. Comunicar, 33 (XVII), 165-174.

LATORRE, A., DEL RINCÓN, D. \& ARNAL, J. (2003). Bases metodológicas de la investigación educativa. Barcelona: Ediciones Experiencia

LIM, D. H., MORRIS, M. L., \& KUPRITZ, V. (2007). Online vs. blended learning: Differences in instructional outcomes and learner satisfaction. Journal of Asynchronous Learning Network, $11(2), 27-42$.

MARQUÉS GRAELLS, P. (2005). Las Webs Docentes. Disponible On-Line en: http://dewey.uab.es/pmarques/webdocente.htm [Consultado en Noviembre de 2010].

MARTínEZ-MIGUÉLEZ, M. (2004) Los Grupos Focales de Discusión como Método de Investigación. Heterotopía, 26, 59-72.

MONEDERO, J.J. (2007). El diseño de los materiales educativos ante un nuevo reto en la enseñanza universitaria: el Espacio Europeo de Educación Superior (EEES). Revista Interuniversitaria de Formación del Profesorado, 21, (1), 51-68.

MUR, F. \& SERRANO, C. (2006). Elaboración de una web docente. 5campus.org. Disponible On-Line en: http://www.5campus.org/leccion/webdocente [Consultado en Octubre de 2010].

RECIO, M.A. \& CABERO, J. (2005). Enfoques de aprendizaje, rendimiento académico y satisfacción de los alumnos en formación en entornos virtuales. Pixel-Bit. Revista de Medios y Educación, 25, 93-115.

SALINAS, J.M. (1997) Enseñanza flexible, aprendizaje abierto. Las redes como herramientas para la formación. Revista Electrónica de Tecnología Educativa, 10, 02/99. Disponible OnLine en: http://www.uib.es/depart/gte/edutec-e/revelec10/revelec10.html. [Consultado en Noviembre de 2007].

SHAHZAD, A.H. \& KHAN, A. (2010). Virtual learning and students perception-a research study. Procedia Social and Behavioral Sciences, 2, 5463-5467.

TESONE, D.V. \& RICCI, P. (2008). Student perceptions of Web-based instruction: a comparative análisis. MERLOT Journal of Online Learning and Teaching, 4 (3), 317-324.

VILLA, O. (2005). La satisfacción de los alumnos en cursos on-line. Revista EducaWeb. Disponible On-Line en: http://www.educaweb.com/noticia/2005/09/26/satisfaccionalumnos-cursos-on-line-1676.html. [Consultado en Enero de 2010]. 
Para citar este artículo:

MIRETE, A. B.; GARCÍA, F. A.; SÁNCHEZ, M. C. (2011). Implicación del alumnado en la valoración de su satisfacción con las web didácticas. Edutec-e, Revista Electrónica de Tecnología Educativa, 37. Recuperado el dd/mm/aa de http://edutec.rediris.es/revelec2/revelec37/implicacion_alumnado_valoracion_satisfacion_ webs_didacticas.html 